\title{
DEPRESSÃO EM PACIENTES COM FOBIA SOCIAL
}

\author{
Depression in Patients with Social Phobia
}

\author{
Gustavo J. Fonseca D’El Rey ${ }^{1}$ \\ Jonathan Joseph Freedner ${ }^{2}$
}

\section{Resumo}

A fobia social (também conhecida como transtorno de ansiedade social) é um dos transtornos mentais mais prevalentes na população geral. A depressão maior é freqüentemente uma complicação na fobia social (generalizada e circunscrita). Os autores neste estudo examinaram retrospectivamente o curso longitudinal da depressão maior em 63 pacientes com diagnóstico de fobia social, por meio da Entrevista Clínica Estruturada para o DSM-IV (versão clínica). Trinta e cinco por cento dos pacientes apresentaram história de pelo menos um episódio depressivo maior. Nesta amostra, os pacientes com fobia social generalizada e pacientes com fobia social circunscrita apresentaram porcentagens semelhantes de depressão maior (37\% e $30 \%$, respectivamente). Os pacientes com maior incapacitação em seu funcionamento pessoal devido à fobia social apresentaram mais transtomos depressivos. As implicações clínicas destes achados são discutidas dentro do contexto atual do desenvolvimento de sintomas depressivos em pacientes com transtornos de ansiedade. Também, as implicações para o tratamento psicológico (intervenções cognitivo-comportamentais) e farmacológico são brevemente discutidas em pacientes com fobia social e depressão maior concomitante.

Palavras-chaves: Fobia social; Depressão; Comorbidade; Curso longitudinal.

\section{Abstract}

Social phobia (also known as social anxiety disorder) is the most prevalent mental disorders in the general population. The major depression is frequently a complication in the social phobia (generalized and specific). The authors in this study examined the longitudinal course of major depression retrospectively in 63 patients with diagnostic of social phobia, by the Structured Clinical Interview for the DSM-IV (clinical version). Thirty five percent of the patients had experienced at least one major depression episode. In this sample patients with generalized social phobia and patients with specific social phobia had comparable past rates of major depression (37\% and 30\%, respectively). The patients with more impairment motived for the social phobia, had more depressive disorders. The clinical implications of these findings are discussed within of the actual context of the development of depressive symptoms in patients with anxiety disorders. Also, the implications for the psychological (cognitive-behavioral interventions) and pharmacological treatments are briefly discussed in patients with social phobia and major depression concomitant.

Keywords: Social phobia; Depression; Comorbidity; Longitudinal course.

1 Psicólogo. Especialista em Psicologia Hospitalar pela Faculdade de Medicina da Universidade de São Paulo. Aprimoramento em Psicopatologia pela Faculdade de Psicologia da Universidade São Judas Tadeu. Coordenador do Programa de Fobia Social do Centro de Pesquisas e Tratamento de Transtornos de Ansiedade - São Paulo-SP, Brasil. Endereço para contato: Rua Bom Jesus, 274-B. São Paulo-SP. CEP 03344-000.

E-mail: g.delrey@bol.com.br

2 Psicólogo. Ph.D. em Psicologia Clínica pela Temple University. Professor da Adult Anxiety Clinic of Temple University, Departament of Psychology, Temple University - USA. 


\section{Introdução}

A fobia social (também chamada de transtorno de ansiedade social) é um transtorno mental severo que traz sofrimento e perdas de oportunidades para seus portadores. Muitas pessoas convivem com este transtorno por anos e sem tratamento adequado em saúde mental, ficando desta maneira sujeitos às privações e sofrimento que 0 transtorno traz e às comorbidades freqüentes que a fobia social carrega (D’El Rey \& Pacini, 2005 e Lamberg, 1998).

Atualmente sabe-se que a depressão é uma comorbidade freqüente dos transtornos de ansiedade. Este tipo de comorbidade tem conseqüências importantes para o diagnóstico, tratamento e prognóstico dos transtornos ansiosos (Chartier, Walker \& Stein, 2003).

Uma grande parte dos estudos se concentra na comorbidade do transtorno de pânico com a depressão. Como a fobia social até poucos anos atrás era considerada como um transtorno de ansiedade negligenciado pelos profissionais de saúde mental, infelizmente existem poucos estudos sobre a comorbidade entre a fobia social e os transtornos depressivos (Lamberg, 1998; Stein \& Kean, 2000).

A maior parte dos estudos de comorbidade entre a fobia social e a depressão se concentra em amostras epidemiológicas e não em amostras clínicas (Magee, Eaton, Wittchen, McGonagle \& Kessler, 1996 e Davidson, Hughes, George \& Blazer, 1993).

Até onde sabemos, existem poucos estudos aqui no Brasil sobre a incidência de transtornos depressivos em pacientes com fobia social, um dos estudos é o de Versiani \& Nardi (1994), que encontraram 29,6\% de depressão maior e 18,4\% de distimia em uma amostra de 250 pacientes com diagnóstico de fobia social.

\section{Objetivo}

Este trabalho tem como objetivo verificar a comorbidade e o curso longitudinal da depressão maior na fobia social em uma amostra clínica de 63 pacientes que procuraram tratamento no Centro de Pesquisas e Tratamento de Transtornos de Ansiedade - São Paulo-SP, Brasil com diagnóstico de fobia social.

\section{Metodologia}

A presente pesquisa foi realizada no período de outubro de 2004 a novembro de 2005, com pacientes de ambos os sexos e maiores de 18 anos do Programa de Fobia Social do Centro de Pesquisas e Tratamento de Transtornos de Ansiedade - São Paulo-SP que no momento da entrevista inicial preencheram os critérios diagnósticos do DSM-IV para fobia social. Este centro de tratamento e pesquisa é composto por psicólogos e psiquiatras e visa ao tratamento de transtornos de ansiedade. Existem 4 programas, ou seja, o Programa de Fobia Social, o Programa de Pânico, o Programa de Transtorno Obsessivo-Compulsivo e o Programa de Estresse Pós-Traumático. Duas orientações norteiam os trabalhos, uma cognitivo-comportamental e outra biológica. Os pacientes foram encaminhados para o referido centro por profissionais de saúde mental da cidade de São Paulo-SP ou vieram até o local de tratamento por anúncios em jornais locais.

Os pacientes deste estudo foram avaliados pelo primeiro autor pelos critérios diagnósticos para fobia social contidos na Entrevista Clínica Estruturada para o DSM-IV - SCID (versão clínica), e dos critérios para depressão maior contidos no mesmo instrumento. A SCID-CV traduzida e adaptada mostrou ser um instrumento diagnóstico confiável e adequado para o uso no Brasil (DelBem et al., 2001). Durante as entrevistas, também se avaliou o grau de funcionamento atual dos pacientes com fobia social. Para isso, utilizou-se a Escala de Impressão do Funcionamento Global (Cooper, 1963), que avalia o grau de interferência de sintomas psicopatológicos no funcionamento do paciente. Este instrumento é baseado em uma escala de 5 pontos ( $1=$ sem sintomas; $2=$ alguns sintomas estão presentes, mas eles não interferem no trabalho ou atividades sociais; $3=$ sintomas interferem um pouco no trabalho ou em atividades sociais; $4=$ sintomas interferem muito no trabalho e atividades sociais; e $5=$ as atividades de trabalho e sociais estão seriamente comprometidas pelos sintomas).

A idade de início da fobia social foi determinada pela recordação do paciente de quando os sintomas fóbicos começaram.

Para a análise estatística, utilizaram-se cálculos de porcentagens, médias, desvios padrões e o teste do qui-quadrado (McCall, 1990). 
Em relação aos procedimentos éticos, 0 paciente recebia um formulário contendo os objetivos da pesquisa, bem como o consentimento informado para sua participação. Não ocorreram recusas na participação.

\section{Resultados}

Neste estudo, foram avaliados 63 pacientes, sendo 24 (38\%) homens e 39 (62\%) mulheres, com idades entre 18 e 56 anos, sendo a média de 36,5 anos. As características sociodemográficas dos pacientes deste estudo estão sumarizadas na Tabela 1 para uma melhor visualização.

\section{Tabela 1- Características da a mostra clínica.}

Características

Sexo

Estado marital

$\begin{array}{ll}\text { Homens (n) } & 24 \\ \text { Porcentagem } & 38 \% \\ \text { Mulheres (n) } & 39 \\ \text { Porcentagem } & 62 \%\end{array}$

Idade (anos)

$\begin{array}{ll}\text { Solteiro (n) } & 28 \\ \text { Porcentagem } & 44 \% \\ \text { Casado (n) } & 35 \\ \text { Porcentagem } & 56 \%\end{array}$

Idade de início (anos)

Média

D.V.

Duração da fobia (anos)

Média 21,0

D.V.

Funcionamento global atual

$\begin{array}{ll}2-3 \text { pontos }(\mathrm{n}) & 6 \\ \text { Porcentagem } & 10 \% \\ 4-5 \text { pontos }(\mathrm{n}) & 57 \\ \text { Porcentagem } & 90 \%\end{array}$

Vinte e dois (35\%) dos 63 pacientes com fobia social preencheram em algum momento de suas vidas também os critérios diagnósticos do DSM-IV para depressão maior. Os pacientes foram subdivididos em fobia social generalizada ( $n=43$ ) e fobia social circunscrita ( $\mathrm{n}=20$ ). Foi considerada fobia social circunscrita quando o paciente apresentava um ou dois medos sociais, geralmente de desempenho (falar em público, escrever diante de outros, etc.). E foi considerada como generalizada o quadro que envolvia diversas situações sociais. Foi hipotetizado que os pacientes com fobia social generalizada apresentariam mais comorbidade com a depressão do que os pacientes com a forma circunscrita. Entretanto, estes dois subgrupos não diferiram em termos de comorbidade com a depressão maior, ou seja, 16 (37\%) dos 43 pacientes com fobia social generalizada preenchiam também os critérios para depressão maior e 6 (30\%) dos 20 pacientes com fobia social circunscrita $\left(\mathrm{x}^{2}=0,31\right)$.

A relação temporal entre o início da fobia e o início do primeiro episódio depressivo maior foi examinada nos pacientes com fobia social e história de depressão maior ( $\mathrm{n}=$ 22). Um (5\%) dos 22 pacientes com fobia social e história de depressão maior teve seu primeiro episódio depressivo 11 anos após o início da fobia social, e um outro paciente (5\%) teve seu primeiro episódio depressivo ocorrendo conjuntamente com o início da fobia social. Vinte (90\%) dos 22 pacientes tiveram seu primeiro episódio depressivo maior entre 1 e 5 anos após o início da fobia social.

Em relação à Escala de Impressão do Funcionamento Global nos pacientes com fobia social e depressão maior $(n=22)$, apenas um (5\%) paciente com fobia social e história de depressão estava dentro do escore entre 2 3 pontos, ou seja, 21 (95\%) pacientes com fobia social e depressão maior estavam dentro do escore entre 4 - 5 pontos. A Tabela 2 apresentará estes resultados para uma melhor visualização.

\footnotetext{
Nota: $\quad$ D.V. $=$ Desvio padrão.
} 
Tabela 2- Funciona mento Global ( $\mathrm{n}=$ 22).

$\begin{array}{cc}\text { Escores } & \text { n (\%) } \\ 1 \text { ponto } & 0(0 \%) \\ 2 \text { pontos } & 0(0 \%) \\ 3 \text { pontos } & 1(5 \%) \\ 4 \text { pontos } & 13(59 \%) \\ 5 \text { pontos } & 8(36 \%)\end{array}$

\section{Discussão}

Neste estudo, encontramos 35\% da amostra de pacientes com fobia social com histórias de episódios depressivos maiores. Este nosso achado é similar ao encontrado por Versiani \& Nardi (1994), ou seja, 29,6\% dos pacientes com fobia social apresentavam depressão maior concomitante.

Os achados deste estudo devem ser interpretados exclusivamente em termos de uma amostra clínica e não epidemiológica. Infelizmente nosso estudo tem algumas limitações metodológicas (ex.: amostra clínica é menor do que amostra epidemiológica, o estudo foi retrospectivo, tínhamos que confiar na lembrança do paciente), porém alguns pontos podem ser destacados.

Por exemplo, nem todos os transtornos de ansiedade estão associados ao mesmo grau de incapacitação no funcionamento pessoal. Os pacientes com fobia social estão mais severamente incapacitados em termos de funcionamento pessoal no dia-a-dia (ver Tabela 1), por isso provavelmente estejam em maior risco para desenvolverem quadros depressivos, como afirmam Chartier et al. (2003), Stein \& Ken (2000) e Lipsitz \& Schneier (2000).

Moscovitch, Hoffmann, Michael \& In-Albon (2005) \& Klein (1999) propõem que os pacientes com fobia social geralmente desenvolvem quadros depressivos como conseqüência direta da desmoralização que eles experienciam como resultado de viverem com ansiedade crônica e limitações fóbicas. Klein (1999) refere-se e estes quadros depressivos como "síndrome desmoralizante".

A associação entre fobia social e depressão maior nos leva a hipotetizar que a fobia social e depressão são variantes de uma mesma doença que aparece em tempos diferentes; o estado comórbido representa uma terceira identidade nosológica; e a fobia social e a depressão são doenças distintas. As investigações disponíveis até o momento não nos permitem chegar a uma conclusão válida a este respeito, como afirmam Chaterjee, Sunitha \& Chartier (1997).

A correta identificação de síndromes depressivas em pacientes com fobia social tem implicações importantes para o tratamento do quadro ansioso (Chartier et al., 2003). Por exemplo, em relação ao tratamento farmacológico, pacientes com fobia social que tem história de depressão prévia podem apresentar sintomas depressivos com o uso de benzodiazepínicos (Gould, Buckminster, Pollack \& Yap, 1997). Em relação ao tratamento psicológico, as implicações também são grandes, pacientes com fobia social e depressão conjuntamente sentem-se desmotivados a aderirem a programas de tratamentos cognitivo-comportamentais, uma vez que estes tratamentos requerem a cooperação ativa do paciente, por exemplo, nas tarefas de exposição a situações temidas, na identificação e correção de pensamentos disfuncionais que geram e mantêm a esquiva de situações sociais, etc. (Lincoln etal., 2003 \& Heimberg, 2001). Existe ainda uma outra complicação na psicoterapia de pacientes com fobia social e depressão concomitante. Os estudos realizados por estudiosos como Heimberg (2001), Heimberg, Salzman, Holt \& Blendell, (1993) mostram que as terapias de grupo são superiores as individuais no tratamento da fobia social, como na depressão o paciente apresenta geralmente uma apatia e falta de interesse em atividades grupais, as terapias grupais de base cognitivo-comportamentais geralmente costumam falhar em pacientes fóbicos sociais com depressão comórbida (Lincoln et al., 2003 e Chambles, Tran \& Glass, 1997).

A fobia social é agora reconhecida como um transtorno altamente incapacitante. Entretanto a maioria dos pacientes não procura tratamento especializado. Isto ocorre também com as síndromes depressivas, na qual um alto grau de incapacitação é acompanhado de ausência de tratamento (Chartier et al., 2003 \& Lamberg, 1998). Adicionando os dois diagnósticos (fobia social e depressão maior), certamente nos confrontamos com uma subamostra de indivíduos que deve ser prontamente diagnosticada e tratada.

Como a depressão é uma complicação freqüente da fobia social, o inverso também deve 
ser verdadeiro, ou seja, pacientes que procuram tratamento para quadros depressivos deveriam ser investigados sistematicamente quanto à presença de ansiedade social no desencadeamento de síndromes depressivas, principalmente em crianças e adolescentes, como descrevem Strauss \& Last (1993).

\section{Considerações Finais}

Podemos concluir neste estudo, que pacientes com fobia social têm um alto índice de história de depressão maior concomitante. $\mathrm{O}$ intervalo entre o início da fobia social e o desenvolvimento de síndromes depressivas neste estudo foi de 1 a 5 anos. Os pacientes mais severamente incapacitados pela fobia social apresentam uma maior chance de apresentarem depressão. A correta identificação de quadros depressivos em fóbicos sociais tem implicações importantes para o sucesso de tratamentos psicológicos (terapia cognitivo-comportamental) e farmacológicos.

Sugerimos que outros trabalhos semelhantes sejam realizados em nosso país, para uma maior confirmação dos dados por nós encontrados.

\section{Agradecimentos}

Gostaríamos de agradecer ao Dr. Richard G. Heimberg do Departamento de Psicologia Temple University - USA, pelas sugestões ao longo deste trabalho.

\section{Referências}

Chambles, D. L, Tran, G. Q., \& Glass, C. R. (1997). Predictors of response to cognitive-behavioral group therapy for social phobia. Journal of Anxiety Disorders, 11(2), 211-240.

Chartier, M. J., Walker, J. R., \& Stein, M. B. (2003). Considering comorbidity in social phobia. Social Psychiatry and Psychiatric Epidemiologic, 38(12), 728-734.

Chaterjee, S., Sunitha, T. A., \& Chartier, M. J. (1997). An investigation into the psychobiology of social phobia: personality domains and seretonergic function. Acta Psychiatrica Scandinava, 95(5), 544550.
Cooper, J. E. (1963). A study of behaviour therapy in 30 psychiatric patients. Lancet, 1(5), 411-415.

Davidson, J. R. T., Hughes, D. C., George, L. K., \& Blazer, D. G. (1993). The epidemiology of social phobia: findings from the Duke Epidemiological Catchement Area Study. Psychological Medicine, 23(4), 709-718.

Del-Ben, C. M., Vilela, J. A. A., Crippa, J. A. S., Hallack, J. E. C., Labate, C. M., \& Zuardi, A.W. (2001). Confiabilidade da "Entrevista clínica estruturada para o DSM-IV - versão clínica" traduzida para o português. Revista Brasileira de Psiquiatria, 23(3), 156-159.

D’El Rey, G. J. F., \& Pacini, C. A. (2005). Tratamento da fobia social por exposição ao vivo e reestruturação cognitiva. Revista de Psiquiatria Clínica, 32(4), 231-235.

Gould, R. A., Buckminster, A., Pollack, P. M., \& Yap, R. T. (1997). Cognitive-behavioral and pharmacological treatment for social phobia: a metaanalysis. Clinical Psychology: Science and Practice, 4(3), 296-306.

Heimberg, R. G. (2001). Current status of psychotherapeutic interventions for social phobia. Journal of Clinical Psychiatry, 62(1), 36-42.

Heimberg, R. G., Salzman, D. G., Holt, C. S., \& Blendell, K. A. (1993). Cognitive-behavioral group treatment for social phobia: effectiviness at five-years follow-up. Cognitive Therapy and Research, 17(3), 325-339.

Klein, D. F. (1999). Anxiety reconceptualized. In: D. F. Klein \& J. Rabkin (Org.). Anxiety: new research and changing conce pts ( $7^{\text {th }}$ ed.). (pp. 218-234). New York: Raven Press.

Lamberg, L (1998). Social phobia: not just another name for shyness. Journal of American Medical Academy, 280(8), 685-686.

Lincoln, T. M., Rief, W., Hahlweg, K., Frank, M., Schroeber, B., \& Fiegenbaum, W. (2003). Effectiveness of an empirically supported treatment for social phobia in the field. Behaviour Research and Therapy, 41(11), 1251-1269.

Lipsitz, J. D., \& Schneier, F. R. (2000). Social phobia epidemiology and cost of illness. Pharmacoeconomics, 18(1), 23-32. 
Magee, W. J., Eaton, W. W., Wittchen, H-U., McGonagle, K. A., \& Kessler, R. C. (1996). Agoraphobia, simple phobia, and social phobia in the National Comorbidity Survey. Archives of General Psychiatry, 53(2), 159-168.

McCall, R. B. (1990). Fundamental statistics for the behavioral sciences $\left(5^{\text {th }}\right.$ ed). New York: Harcourt B. Jovanovich.

Moscovitch, D. A., Hoffmann, S. G., Michael, K., \& In-Albon, T. (2005). Mediation of changes in anxiety and depression during treatment of social phobia. Journal of Consulting and Clinical Psychology, 73(5), 945-952.
Stein, M. B., \& Kean, Y. M. (2000). Disability and quality of life in social phobia: epidemiologic findings. American Journal of Psychiatry, 157(10), 1606-1613.

Strauss, C. C., \& Last, C. G. (1993). Social and simple phobias in children. Journal of Anxiety Disorders, 7(2), 141-152.

Versiani, M., \& Nardi, A. E. (1994). Social phobia and depression. Depression and Anxiety, 5(2), 28-32. 